\title{
Les jeunes atteints de maladie mentale : répondre au besoin de services
}

$\mathrm{L}$ es troubles mentaux constituent collectivement le fardeau de maladie le plus lourd dans le monde. Chez les 15 à 44 ans, par exemple, quatre des cinq maladies les plus invalidantes sont des troubles mentaux : dépression, trouble bipolaire, abus d'alcool et schizophrénie ${ }^{1}$. Ces troubles se caractérisent par une maladie grave et la mort prématurée, et ont une incidence sur l'économie. Outre le trouble d'hyperactivité avec déficit de l'attention, les troubles du développement omniprésents et les démences qui constituent les maladies mentales persistantes les plus courantes ont tendance à manifester leurs premiers symptômes au cours des Io à 15 années qui suivent la puberté. Au Canada, de $14 \%$ à $25 \%$ des jeunes sont aux prises avec un trouble mental. Le taux de suicide grimpe rapidement pendant cette période.

Dans l'ensemble, les jeunes sont en bonne santé physique. Leur proportion de l'utilisation des ressources coûteuses du système de santé (c.-à-d. lits en service interne) est attribuable pour la plus grande part aux maladies mentales. D'autres problèmes de santé courants tels que l'abus de substances et les accidents sont souvent reliés à des troubles mentaux. Ces problèmes ont un effet sur les familles, les pairs, les écoles et les communautés.

On a démontré que l'identification précoce accompagnée du diagnostic et du traitement appropriés peuvent lutter efficacement contre les maladies mentales chez les jeunes dans des contextes de soins primaires et de soins spécialisés. Les interventions appropriées au cours de la jeunesse peuvent réduire l'incapacité et améliorer la réussite professionnelle et la qualité de vie. L'intervention précoce au moyen de thérapies efficaces pourrait ainsi améliorer considérablement la santé de la population dans son ensemble en même temps que les perspectives d'avenir des jeunes en cause.

Compte tenu de cette juxtaposition du besoin et de la capacité de répondre efficacement à ce besoin, il serait raisonnable de s'attendre à ce que les services de santé au Canada soient organisés de façon à optimiser l'interface entre les services et les besoins chez les jeunes atteints de maladie mentale. Or, ce n'est malheureusement pas le cas. Au moment même où les jeunes ont le plus besoin de soins de santé mentale accessibles et dispensés de façon efficace, il arrive souvent que ces services ne soient pas disponibles. Les milieux de soins de santé primaires sont mal équipés pour répondre à ces besoins. La formation, le perfectionnement professionnel et le soutien continu des psychiatres spécialisés en santé des enfants et des adolescents ne suffisent pas. Quant aux soins de santé mentale spécialisés, ils sont répartis entre les services aux enfants et aux adultes : souvent, les jeunes ne sont ni désirés en soins pédiatriques ni bien accueillis en soins aux adultes.

En dehors du système de santé, il existe tout un éventail d'organisations non gouvernementales qui fournissent aux jeunes des services de santé mentale. On ne sait pas cependant si ces prestateurs possèdent les compétences nécessaires pour bien identifier les jeunes atteints de maladie mentale et intervenir de façon appropriée. De nombreux programmes scolaires et communautaires de "prévention» lancés avec enthousiasme ont fait leur apparition récemment à l'intention de jeunes en détresse mentale. Malheureusement, il n'existe guère de données scientifiques valides pour démontrer leur efficacité dans la prévention des maladies mentales. Entre-temps, comparativement à la pléthore de nouveaux programmes à l'intention des jeunes en détresse, on affecte peu de ressources thérapeutiques, voire même aucune, aux besoins de ceux qui sont atteints de troubles mentaux complexes et persistants.

Les années d'adolescence sont caractérisées par des transformations importantes et rapides de tout un éventail de dimensions sociales, personnelles, cognitives et comportementales. Ces changements découlent de l'interaction complexe entre le développement rapide du cerveau et les défis environnementaux qui surgissent pendant cette période. Les interventions en soins de santé mentale qui visent cette population doivent être éclairées et orientées par les besoins du développement - physiologique, psychologique et social. Compte tenu du fardeau imposé par les troubles mentaux diagnosticables et traitables qui se manifestent au cours de l'adolescence, il est essentiel de disposer de soins de santé mentale factuels et appropriés pour répondre à ces besoins. Les ressources devraient intégrer horizontalement tout un éventail d'interventions dans les services de santé actuels.

Il est injuste et inéquitable que les stratèges du système de santé et les prestateurs de soins continuent de contourner ou d'ignorer ces besoins. Dans son récent rapport ${ }^{4}$ intitulé De l'ombre à la lumière : la transformation des services concernant la santé mentale, la maladie mentale et la toxicomanie au Canada, le Sénat a enfin insisté sur la nécessité pour les Canadiens de répondre aux besoins des personnes atteintes de maladie mentale. Or, ces besoins sont les plus criants chez nos jeunes.

Dans aucun autre groupe les investissements ne sont-ils susceptibles de produire de plus grands résultats.

\section{Stan Kutcher}

Titulaire de la chaire de la Financière Sun Life en santé mentale des adolescents

Directeur, Centre de collaboration de l'Organisation mondiale de la santé et de l'Organisation panaméricaine de la santé en politique et formation en santé mentale, Université Dalhousie Halifax (N.-É.)

\section{Simon Davidson}

Directeur, Division de la psychiatrie de l'enfant et de l'adolescent Département de psychiatrie, Université d'Ottawa

Directeur général, Planification et développement

Centre d'excellence provincial en santé mentale des enfants et des adolescents

Centre hospitalier pour enfants de l'est de l'Ontario, Ottawa (Ont.)

Cet article a fait l'objet d'un examen par les pairs.

\section{RÉFÉRENCES}

I. Organisation mondiale de la santé. Rapport sur la santé dans le monde 200I. La santé mentale : Nouvelle conception, nouveaux espoirs. Genève : L'Organisation; 200I. Disponible : www.who.int/entity/whr/200I/en/whroI_fr.pdf (consulté le II janvier 2007)

2. Waddell C, Offord DR, Shepherd CA, et al. Child psychiatric epidemiology and Canadian public policy-making: the state of the science and the art of the possible. $R$ Can Psychiatrie 2002;47:825-32.

3. Santé Canada. Rapport sur les maladies mentales au Canada. Ottawa : Santé Canada; 2002. No o-662-32817-5. Disponible: www.phac-aspc.gc.ca/publicat /miic-mmac/pdf/men_ill_f.pdf(consulté le ir janvier 2007).

4. Kirby MJL, Keon WJ. De l'ombre à la lumière : la transformation des services concernant la santé mentale, la maladie mentale et la toxicomanie au Canada. Ottawa : Comité sénatorial permanent des affaires sociales, des sciences et de la technologie; 2006. Disponible : www.parl.gc.ca/39/I/parlbus/commbus/senate/com-f/socif/rep-f/repo2mayo6-f.htm (consulté le 4 décembre 2006). 